\title{
Guidance of an Autonomous Surface Vehicle for Underwater Navigation Aid
}

\author{
José P. Sousa $^{\dagger}$, Bruno M. Ferreira*, Nuno A. Cruz ${ }^{* \dagger}$ \\ ${ }^{*}$ INESC TEC, ${ }^{\dagger}$ Faculty of Engineering, University of Porto \\ Rua Dr. Roberto Frias \\ 4200-465 Porto, Portugal \\ up201303822@fe.up.pt
}

\begin{abstract}
Unmanned Underwater Vehicles (UUVs), such as Autonomous Underwater Vehicles (AUVs) and Remotely Operated Vehicles (ROVs) are versatile tools, suitable for many activities in different fields, and have seen an increase in usage, making them an area of interest in the study of robotics. The performance of any underwater vehicle in any given task is deeply affected by the precision of its localization system. The main challenge in underwater localization is the significant attenuation of any Radio Frequency (RF) signal underwater, which prevents the use of many common location methods such as the Global Positioning System (GPS). Many methods have been studied for the localization of UUVs, including the use of acoustic beacons. One of these methods is the use of a single moving beacon to obtain acoustic ranges, as opposed to a stationary single beacon, which restricts the UUV's trajectory or multiple beacons, involving more hardware, complicating missions' logistics and increasing costs.

In this paper, a guidance algorithm based on the Fisher Information Matrix is proposed for an Autonomous Surface Vehicle to serve as a beacon vehicle and aid in the navigation of a UUV. The approach performances are assessed by means of simulations of the complete system under realistic conditions.
\end{abstract}

\section{INTRODUCTION}

Unmanned Underwater Vehicles (UUVs), such as Autonomous Underwater Vehicles (AUVs) and Remotely Operated Vehicles (ROVs) are versatile tools, suitable for many activities in different fields, from monitoring underwater pipelines and communication lines to military purposes. The performance of any underwater vehicle in any given task is deeply affected by the accuracy of its localization system. The main challenge in underwater localization is the significant attenuation of any Radio Frequency (RF) signal underwater, which prevents the use of many common localization methods such as the Global Positioning System (GPS)[1]. In order to overcome this difficulty, multiple localization techniques have been developed, based on different technologies, as reviewed in [2]. Cooperative Navigation (CN) is one of these techniques.

In $\mathrm{CN}$, a UUV determines its position relatively to one or more vehicles that can determine their own positions with less uncertainty. These vehicles are commonly referred to as Communication and Navigation Aid (CNA) vehicles.

CN localization methods using only one support vehicle are appealing, to lower costs and logistics, but they require good positioning of the CNA vehicle to maintain observability and achieve good performances [3], [4]. This paper studies metrics based on the Fisher Information Matrix (FIM) to guide a support Autonomous Surface Vehicle (ASV) acting as a CNA in order to improve the localization estimate of an ROV and proposes a guidance algorithm based on the studied metrics.

\section{CONCEPT}

The idea of using only one ASV for underwater localization is not new. The use of one surface craft as a CNA vehicle for a UUV has been studied in [5], and path planning for target tracking using only acoustic range measurements from an ASV has been studied in [6]. This paper proposes a metric for real-time guidance of the surface vehicle.

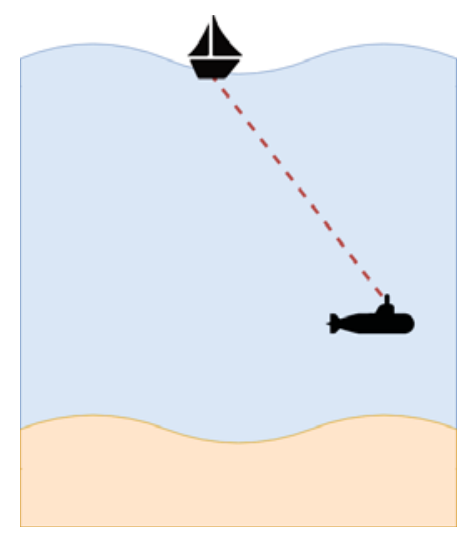

Figure 1: Illustration of the studied concept.

We aim at developing a guidance system for a CNA surface vehicle. In the studied $\mathrm{CN}$ localization system, the UUV estimates its own pose with Dead Reckoning (DR) techniques and periodically obtains ranges from CNA vehicles to improve this estimate, as shown in figure 1, where the red dashed line represents the acoustic ranges. The direction of the obtained ranges is particularly important, hence the need to adapt the surface vehicle's trajectory to the requirements of the UUV. The navigation system developed should be able to determine in real time the trajectory of the CNA that minimizes the position uncertainty of the UUV.

\section{A. Vehicle Overview}

Regarding the ASV, the existence of a localization module is assumed, giving it the ability to determine its position with a small uncertainty (in practice, in the same order of 
magnitude as GPS and IMU devices). It is also assumed that a control module for the vehicle's actuators is also implemented, meaning that the only input needed to move the ASV are the desired linear and angular velocities.

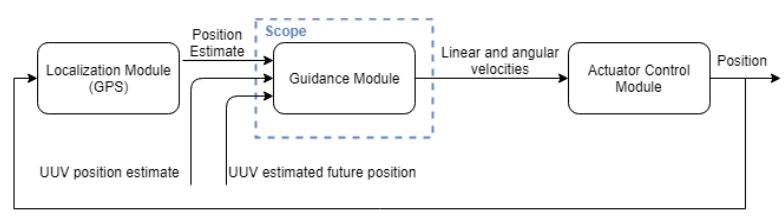

Figure 2: Model for the surface vehicle.

As a result, the scope of this work regarding the ASV, as highlighted in figure 2 , is solely the development of a guidance algorithm that determines the position where the acoustic ranges contribute to minimize the uncertainty of the UUV's position estimate. The inputs for this algorithm are the current position estimate of the ASV, the current position estimate of the UUV and estimates of the future UUV position.

The first assumption made about the underwater vehicle is that it is remotely operated. The scope of this work regarding the ROV is the development of a localization module, as shown in figure 3.

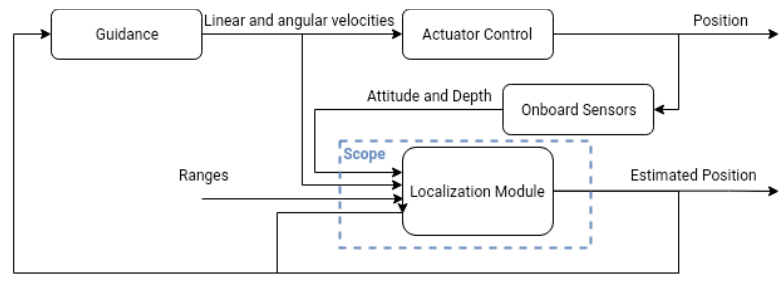

Figure 3: Model of the underwater vehicle.

A control module is assumed to be implemented as was the case with the ASV. Since the vehicle is remotely operated, the guidance is carried out by a human. The inputs for the localization module are the current ROV position estimate, the current ROV speed estimate, current position information (from on board sensors, such as a gyroscope and/or compass) and ranges to the ASV.

\section{Proposed SOLUTION}

This section presents a method for the ROV self-localization built from a standard filter, then proposes ASV guidance approaches to decrease the uncertainty of the ROV localization.

\section{A. Underwater Vehicle Localization}

In order to estimate the position of the ROV, an Extended Kalman Filter was used. The information used in this estimation was the following:

- the vehicle's previous position estimate;

- previous estimate of the vehicle's angular and linear speed;

- measurements of the vehicle's attitude and depth given by onboard sensors;

- range measurements to the ASV.
The transition model used in the prediction step is the one in (1), where $\Delta t$ is the time step.

$$
\begin{gathered}
X(k+1)=f(X(k), \nu(k))+\mathcal{N}(0, Q(k)) \\
f(X(k), \nu(k))=\Delta t * J(X(k)) \cdot \nu(k)
\end{gathered}
$$

Based on this transition model the next state, $\hat{X}(k+1 \mid k)$, alongside the pose's covariance, $P(k+1 \mid k)$ can be predicted.

The next step is the inclusion of measurements given by the onboard sensors, which include measurements of the attitude and depth of the vehicle in order to update the state prediction. Whenever a range measurement is available it must also be incorporated into the state prediction. To accomplish this a second update is made where the expected value and covariance for the next state considering the range measurement are calculated.

\section{B. Surface Vehicle Guidance}

The trajectory of the surface vehicle is waypoint based, that is, waypoints are defined for each moment when an acoustic signal is to be sent. The computation of these waypoints is an iterative process, meaning that these are calculated in real time during the mission. After the surface vehicle sends an acoustic signal, its reaching set is calculated using the amount of time until the next range and its maximum velocity. It is assumed that the surface vehicle is holonomic. In this work, the target point is chosen from the reaching set, according to the metrics described ahead, based on the analysis of the FIM. The use of the FIM as a metric for sensor placement is explored in [7] and [8]. From this point, the subscripts 'ASV' and 'ROV' are used in variables to refer to the surface and underwater vehicle, respectively.

Considering $p=\left[N_{R O V}, E_{R O V}\right]$ as the parameters to estimate, the amount of information that the measurement carries about $p$ can be quantified using the FIM. Assuming Gaussian and independent measurement errors, the information matrix is given by (2). [7]

$$
I(p)=\nabla_{p} Z(p)^{T} Q^{-1} \nabla_{p} Z(p)
$$

The assumption that the ROV is stationary between consecutive range measurements to the ASV in different positions creates a synthetic baseline scenario. The information carried by these measurements about the parameters to be estimated is given by the summation of the information matrices of each measurement. For this equation to be valid, the variance of the measurement errors has to be considered equal for both of the ranges.

$$
I(p)=\frac{1}{\delta^{2}} \sum_{i=1}^{N}\left[\begin{array}{c}
\frac{\left(N_{R O V}-N_{A S V i}\right)^{2}}{Z_{i}^{2}} \\
\frac{\left(N_{R O V}-N_{A S V i}\right)\left(E_{R O V}-E_{A S V i}\right)}{Z_{i}^{2}} \\
\frac{\left(N_{R O V}-N_{A S V i}\right)\left(E_{R O V}-E_{A S V i}\right)}{Z_{i}^{2}} \\
\frac{\left(E_{R O V}-E_{A S V i}\right)^{2}}{Z_{i}^{2}}
\end{array}\right]
$$

Using the FIM, the minimum uncertainty of the estimate $\hat{p}$ of the parameter $p$ based on this measurement can be determined, since the Cramer-Rao Lower Bound $(\mathcal{C})$ is equal to the inverse 
of the FIM $(I) . \mathcal{C}$ defines an uncertainty ellipsoid, the volume of which is a measurement of the uncertainty of $\hat{p}$. The determinant of the information matrix is used as a measure of the volume of the uncertainty ellipse, meaning that maximizing the determinant $\operatorname{det}(I(p))$ minimizes the uncertainty of $\hat{p}$. [7].

With these premises, two metrics were constructed to use in the choice of the waypoints.

1) Approach A: In this approach the next position of the ASV is determined using only the current positions of the ASV and ROV.

$$
\begin{gathered}
\operatorname{det}(I(p))=\frac{1}{\delta^{2}} \sum_{i=1}^{N} \frac{\left(N_{R O V}-N_{A S V i}\right)^{2}}{Z_{i}^{2}} * \\
\sum_{i=1}^{N} \frac{\left(E_{R O V}-E_{A S V i}\right)^{2}}{Z_{i}^{2}}- \\
\left(\sum_{i=1}^{N} \frac{\left(N_{R O V}-N_{A S V i}\right)^{2}\left(E_{R O V}-E_{A S V i}\right)}{Z_{i}^{2}}\right)^{2}
\end{gathered}
$$

The determinant of the information matrix, shown in (4) is then considered as a function of the next position of the surface vehicle, which makes it possible to discover the position where $\operatorname{det}(I(p))$ is maximized and, consequently, the uncertainty of $\hat{p}$ is minimized. A surface plot of the determinant in function of the ASV's next waypoint is shown in figure 4. In this case, the ROV is considered to be in position $(0,0,-20)$ and the ASV's coordinates are $(20,20)$.
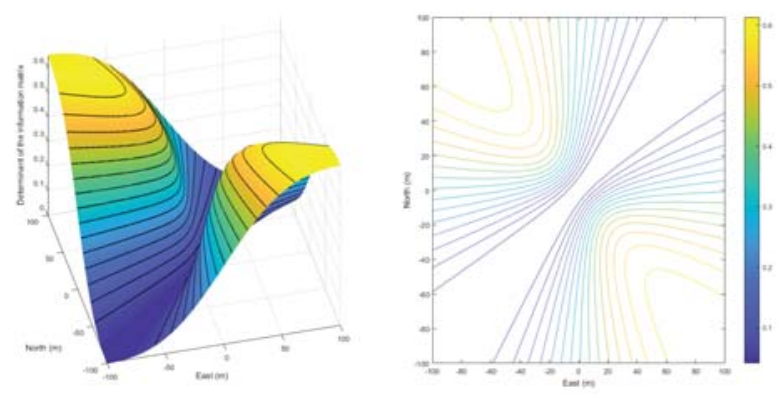

Figure 4: Determinant of the information matrix in function of the ASV's next position.

An example of the behaviour of the guidance algorithm using this metric is shown in figure 5. As previously explained, whenever the surface vehicle (black cross) reaches a waypoint and sends an acoustic signal, the determinant of the information matrix is calculated for all the points within the surface vehicle's reaching set (every point inside the black circumference). The next waypoint is the point among these that maximizes the determinant of the FIM.

2) Approach B: This approach was developed since the assumption that the ROV is stationary is not always true. Since the relative positioning of the ROV and ASV when ranges are sent is a key factor in the uncertainty of $\hat{p}$, the inclusion of an estimate of $X_{R O V 2}$ in this metric may have a positive effect in lowering said uncertainty. This effect is naturally limited by the accuracy of the $X_{R O V 2}$ estimate. A new metric for evaluation of the next ASV position was defined, taking into account the change of the position of the ROV. For that, the current and estimated future positions of the ROV are considered as separate parameters to estimate. These are referred to as $p_{1}=\left(N_{R O V 1}, E_{R O V 1}\right)$ and $p_{2}=\left(N_{R O V 2}, E_{R O V 2}\right)$. To

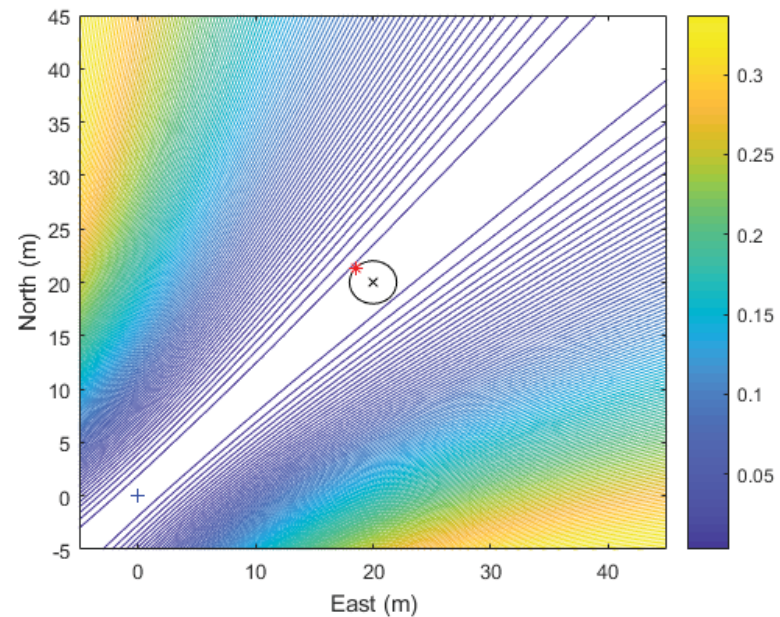

Figure 5: Graphic example of the SV next position.

address this situation, two FIMs are defined. As was the case in the previous approach, the determinant of both matrices are a measurement of the uncertainty of the estimates. The determinants of both matrices are shown in (5).

$$
\begin{gathered}
\operatorname{det}\left(I\left(p_{1}\right)\right)=\frac{1}{\delta^{2}} \sum_{i}^{2} \frac{\left(N_{R O V 1}-N_{A S V i}\right)^{2}}{Z_{i}^{2}} * \\
\sum_{i}^{2} \frac{\left(E_{R O V 1}-E_{A S V i}\right)^{2}}{Z_{i}^{2}}- \\
\left(\sum_{i}^{2} \frac{\left(N_{R O V 1}-N_{A S V i}\right)^{2}\left(E_{R O V 1}-E_{A S V i}\right)}{Z_{i}^{2}}\right)^{2} \\
\operatorname{det}\left(I\left(p_{2}\right)\right)=\frac{1}{\delta^{2}} \sum_{i}^{2} \frac{\left(N_{R O V 2}-N_{A S V i}\right)^{2}}{Z_{i}^{2}} * \\
\sum_{i}^{2} \frac{\left(E_{R O V 2}-E_{A S V i}\right)^{2}}{Z_{i}^{2}}- \\
\left(\sum_{i}^{2} \frac{\left(N_{R O V 2}-N_{A S V i}\right)^{2}\left(E_{R O V 2}-E_{A S V i}\right)}{Z_{i}^{2}}\right)^{2}
\end{gathered}
$$

Since the global objective of this work is to minimize the uncertainty of position of the ROV at all times, the metric defined in this approach is the sum of the determinants, show in (6).

$$
m=\operatorname{det}\left(I\left(p_{1}\right)\right)+\operatorname{det}\left(I\left(p_{2}\right)\right)
$$

For a better understanding of how $m$ varies according to the next waypoint of the ASV, in figure 6 this metric is shown in function of the surface vehicle's next position, considering that the ROV moves from position $[0,0-20]$ to $[20,0,-20]$ and that the current position of the ASV is [20,0].

An example of the behaviour of the guidance algorithm using this metric is shown in figure 7 . Whenever the surface vehicle (black cross) reaches a waypoint and sends an acoustic signal, the metric is calculated for all the points within the surface vehicle's reaching set (every point inside the black circumference). The next waypoint is the point among these that maximizes the metric. 


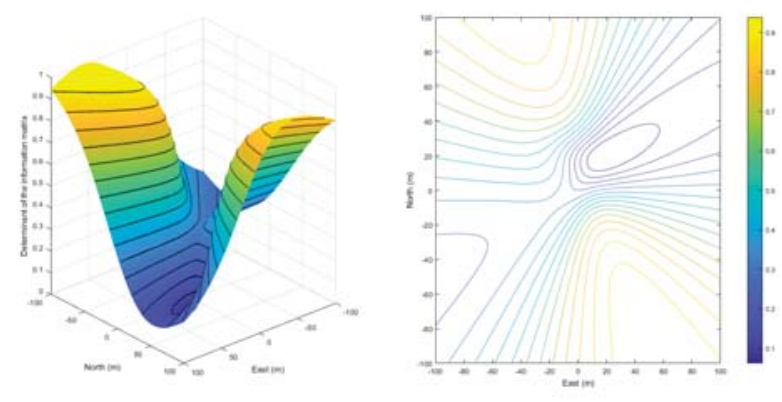

Figure 6: Sum of the determinants in function of the ASV's next position.

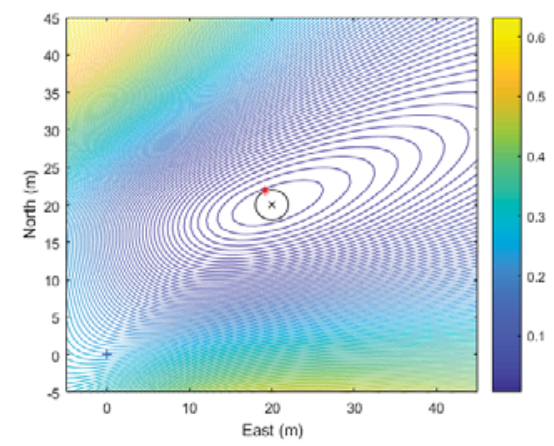

Figure 7: Graphic example of the SV next position.

\section{Simulation Results}

The proposed solution was tested, considering all assumptions made in II, in a simulation environment with the ROV stopped and moving in paths. The different ROV paths used are shown in figure 8 .

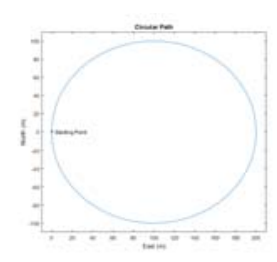

(a) Circular Path

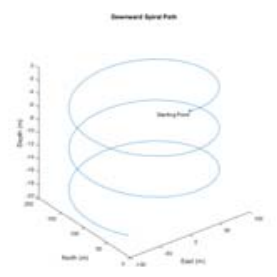

(b) Spiral Path

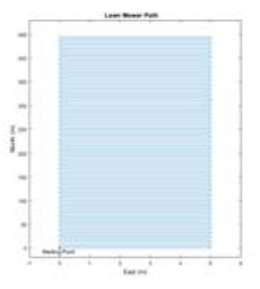

(c) Lawn mower path
Figure 8: Different paths of the ROV used to test the guidance algorithms.

It is important to note that despite the paths being predefined, the guidance of the ASV is performed without using any knowledge of these paths.

The results of the simulations were analyzed in light of the mean and variance of the position estimation error. Since the parameters to estimate are the coordinates of the ROV in the horizontal plane (NE), the estimation error is given by (7).

$$
\varepsilon=\sqrt{\left(N_{R O V}-\hat{N}_{R O V}\right)^{2}+\left(E_{R O V}-\hat{E}_{R O V}\right)^{2}}
$$

The first tests presented are two tests where the starting position of the ROV is the same $(0,0,-5)$ but the path followed is different. It can be clearly seen that when the ROV follows a circular path (9a) the error is bounded, while in the case the path performed is a spiral (9b), the positioning error increases.

Since the main difference between these paths is the increasing depth in the spiral path, the increasing error implicates that the depth of the ROV may have an effect on the positioning error.
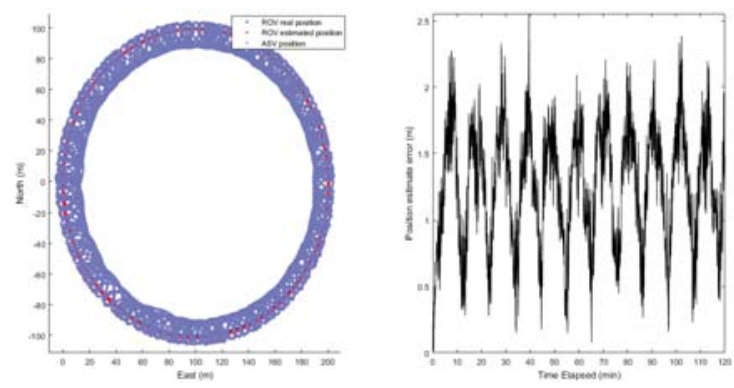

(a) Test with circular path
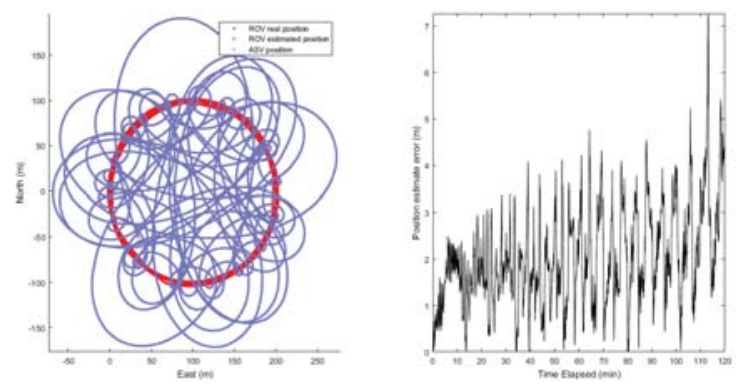

(b) Test with the spiral path

Figure 9: Comparison between two tests with the ROV following the circular and spiral paths.

The tests shown in figure 10, where the starting point of the ASV is $(0,20)$ and the ROV is stationary at two different depths: $(0,0,-5)$ and $(0,0,-20)$.

On the one hand, the fact that the error is greater in $10 \mathrm{~b}$ supports the previous statement that the depth of the ROV has an effect on the estimation error. On the other hand, it is noticeable that the horizontal distance the ASV keeps from the ROV is also different in both scenarios.

The fact that in figure 10a the ASV approaches the ROV until a certain distance and in figure 10b it keeps the original distance indicates that the horizontal distance between the ROV and ASV may also be a factor that influences the error of the estimate.

To infer about the validity of the previous assessments about the influence of the depth of the ROV in the estimation error, an analysis of the metric defined in III-B1 is made.

As mentioned before, the determinant of the information matrix quantifies the uncertainty of the estimation of the horizontal position of the ROV ( $\mathrm{N}$ and $\mathrm{E}$ coordinates) based on two range measurements. After studying the determinant of the information matrix in function of the depth of the ROV and the horizontal distance between the vehicles, it was concluded that the global maximum of determinant of the information matrix decreases with the depth of the ROV and increases with 

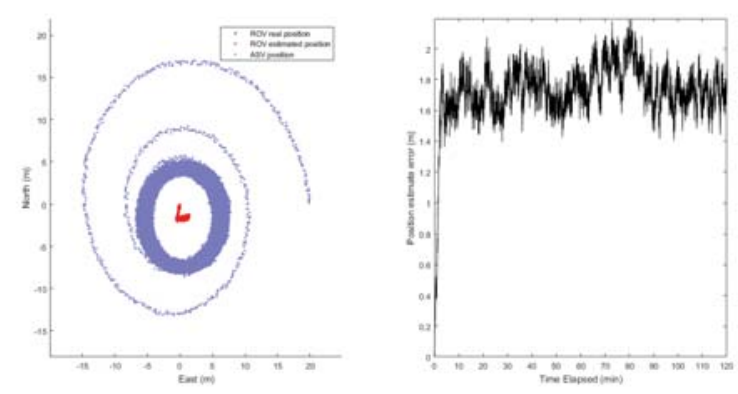

(a) Test with the ROV stationary in $(0,0,-5)$
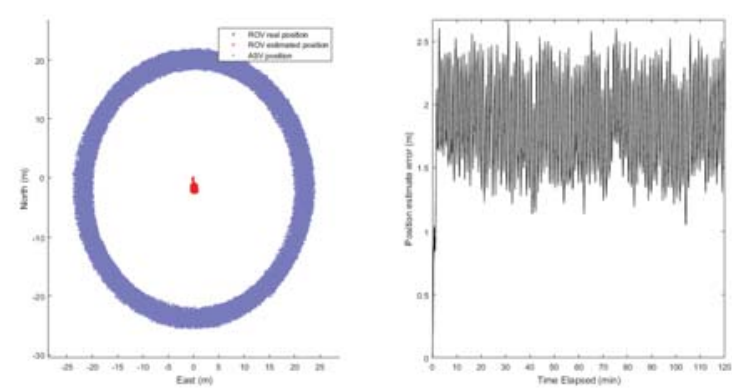

(b) Test with the ROV stationary in $(0,0,-20)$

Figure 10: Comparison between two tests with the ROV stationary at different depths.

the horizontal distance between the ASV and ROV. However, the global maximum determinant of the information matrix is not an indicator of the uncertainty in the obtained estimation. The direct measurement of the minimum uncertainty of the estimate is the maximum determinant within the reaching set of the ASV.

With this knowledge it is possible to conclude that there is a relation between the uncertainty of the estimate in the first pair of ranges, the depth of the ROV, the distance between the ROV and ASV and the speed of the ASV.

Given the existence of this relation, for any given depth of the ROV it is possible to determine a horizontal distance between the vehicles that minimizes the uncertainty for the first two ranges, as long as the reaching set of the ASV is well defined, that is, as long as the time between the ranges and speed of the ASV are known. This position is the one where the determinant of the FIM within the range of the ASV is maximum.

These distances were calculated for different speeds of the ASV and are shown in figure 11. While most ASVs cannot travel as fast as some of speeds shown in the graph, these are presented as a hypothetical scenario.

The maximum determinant of the information matrix for the first two measurements achievable in function of the ROV's depth for different speeds of the ASV is shown in figure 12.

This analysis not only provides an a priori notion of the expected uncertainty for different conditions but also an objective criteria for the initial positioning of the ASV relative to the ROV that minimizes that uncertainty.

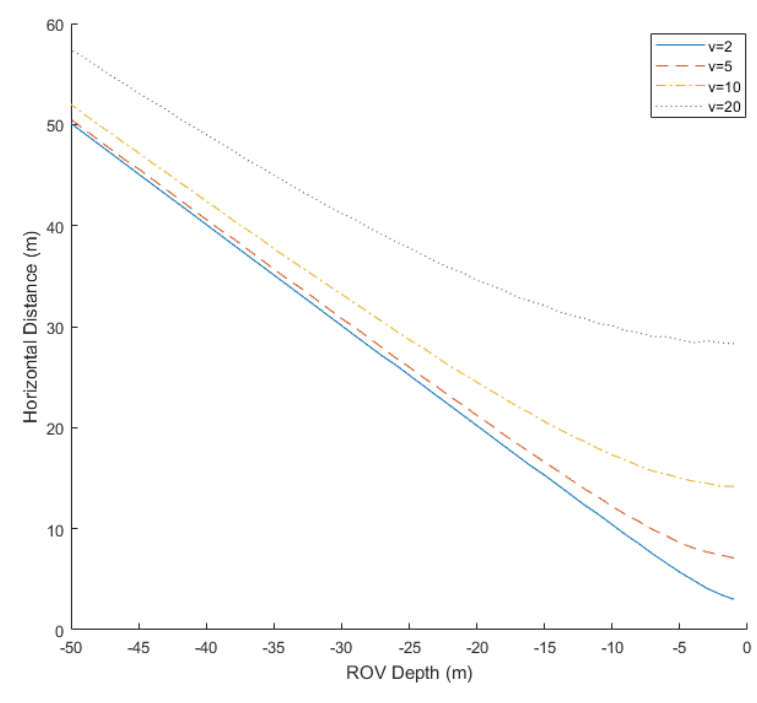

Figure 11: Horizontal distance in function of ROV depth for different speeds of the ASV.

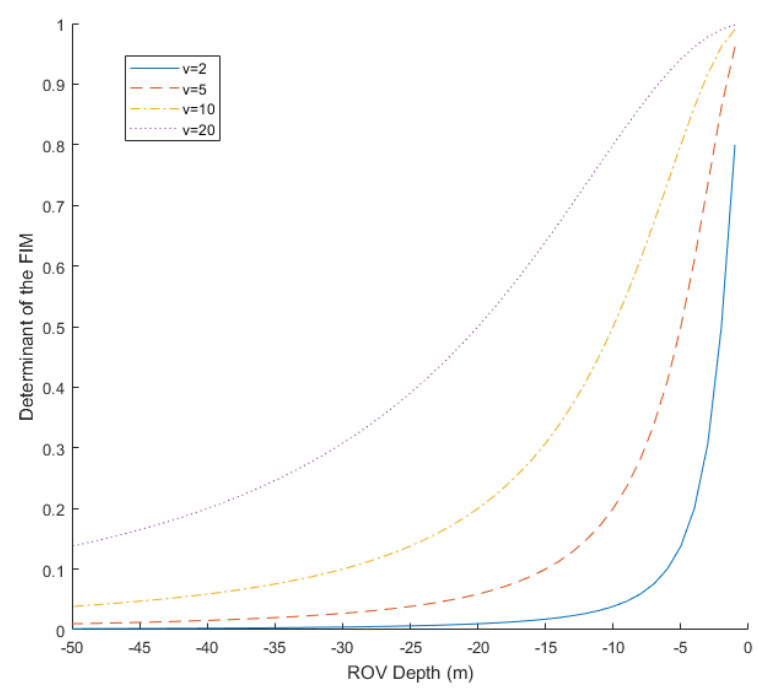

Figure 12: Maximum determinant of the FIM in function of ROV depth for different speeds of the ASV.

\section{A. Approach $A$}

Using this information, the guidance algorithm was tested with the ROV stopped and moving according to the three different paths in figure 8 and the ASV in the optimal starting position shown in figure 11 . The ASV was assumed to have a maximum velocity of $2 \mathrm{~m} / \mathrm{s}$.

The ROV's starting point in the horizontal plane was always $(0,0)$, and various initial depths were tested. The results obtained are shown in table I.

\section{B. Approach B}

The tests made for approach A were also made for approach B. It is considered that no information about the motion of 
Table I: Results of testing approach A with the 4 ROV paths at different depths.

\begin{tabular}{lllll} 
Depth & Stationary & Lawn Mower & Circular & Spiral \\
\hline \multirow{2}{*}{$1 \mathrm{~m}$} & $\bar{\varepsilon}=0.810$ & $\bar{\varepsilon}=0.689$ & $\bar{\varepsilon}=0.575$ & $\bar{\varepsilon}=1.970$ \\
& $\sigma_{\varepsilon}^{2}=0.021$ & $\sigma_{\varepsilon}^{2}=0.016$ & $\sigma_{\varepsilon}^{2}=0.063$ & $\sigma_{\varepsilon}^{2}=1.102$ \\
\hline \multirow{2}{*}{$\mathrm{m}$} & $\bar{\varepsilon}=1,720$ & $\bar{\varepsilon}=1.362$ & $\bar{\varepsilon}=1.255$ & $\bar{\varepsilon}=2.016$ \\
& $\sigma_{\varepsilon}^{2}=0.034$ & $\sigma_{\varepsilon}^{2}=0.043$ & $\sigma_{\varepsilon}^{2}=0.193$ & $\sigma_{\varepsilon}^{2}=1.080$ \\
\hline \multirow{2}{*}{$20 \mathrm{~m}$} & $\bar{\varepsilon}=1.820$ & $\bar{\varepsilon}=1.482$ & $\bar{\varepsilon}=1.488$ & $\bar{\varepsilon}=2.530$ \\
& $\sigma_{\varepsilon}^{2}=0.124$ & $\sigma_{\varepsilon}^{2}=0.361$ & $\sigma_{\varepsilon}^{2}=0.407$ & $\sigma_{\varepsilon}^{2}=1.499$ \\
\hline $50 \mathrm{~m}$ & $\bar{\varepsilon}=1.78$ & $\bar{\varepsilon}=2.309$ & $\bar{\varepsilon}=2.135$ & $\bar{\varepsilon}=3.542$ \\
& $\sigma_{\varepsilon}^{2}=1.213$ & $\sigma_{\varepsilon}^{2}=0.647$ & $\sigma_{\varepsilon}^{2}=0.945$ & $\sigma_{\varepsilon}^{2}=2.228$
\end{tabular}

the ROV is available at the beginning of the mission, so the method used for determining the initial position of the ASV was the same as the one used for approach A, meaning that the only difference between the tests was the guidance algorithm used. As mentioned in section III-B2, this approach includes the motion of the ROV in the guidance of the ASV, by including the estimated position of the ROV at the time the next signal will be sent. The estimated future position of the ROV was calculated under the assumption that the ROV keeps moving in the same direction until the next range. In table II the results of all the tests made using this approach for the guidance algorithm are shown.

Table II: Results of testing approach B with the 4 ROV paths at different depths.

\begin{tabular}{lllll} 
Depth & Stationary & Lawn Mower & Circular & Spiral \\
\hline \multirow{2}{*}{$1 \mathrm{~m}$} & $\bar{\varepsilon}=0.690$ & $\bar{\varepsilon}=0.659$ & $\bar{\varepsilon}=0.537$ & $\bar{\varepsilon}=4.309$ \\
& $\sigma_{\varepsilon}^{2}=0.102$ & $\sigma_{\varepsilon}^{2}=0.014$ & $\sigma_{\varepsilon}^{2}=0.050$ & $\sigma_{\varepsilon}^{2}=6.842$ \\
\hline \multirow{2}{*}{$5 \mathrm{~m}$} & $\bar{\varepsilon}=1,730$ & $\bar{\varepsilon}=1.415$ & $\bar{\varepsilon}=1.170$ & $\bar{\varepsilon}=4.933$ \\
& $\sigma_{\varepsilon}^{2}=0.039$ & $\sigma_{\varepsilon}^{2}=0.043$ & $\sigma_{\varepsilon}^{2}=0.073$ & $\sigma_{\varepsilon}^{2}=6.907$ \\
\hline \multirow{2}{*}{$20 \mathrm{~m}$} & $\bar{\varepsilon}=1.817$ & $\bar{\varepsilon}=1.479$ & $\bar{\varepsilon}=5.637$ & $\bar{\varepsilon}=5.041$ \\
& $\sigma_{\varepsilon}^{2}=0.134$ & $\sigma_{\varepsilon}^{2}=0.207$ & $\sigma_{\varepsilon}^{2}=4.168$ & $\sigma_{\varepsilon}^{2}=4.883$ \\
\hline \multirow{2}{*}{$50 \mathrm{~m}$} & $\bar{\varepsilon}=1.568$ & $\bar{\varepsilon}=2.150$ & $\bar{\varepsilon}=5.074$ & $\bar{\varepsilon}=5.375$ \\
& $\sigma_{\varepsilon}^{2}=1.236$ & $\sigma_{\varepsilon}^{2}=0.478$ & $\sigma_{\varepsilon}^{2}=4.703$ & $\sigma_{\varepsilon}^{2}=3.726$ \\
\hline
\end{tabular}

As mentioned previously, the difference between the two approaches is the inclusion of an estimate of the ROV's future position. It can be deduced that approach B performs better than approach A when the estimation of the ROV's next position is accurate. Since the method used to predict the ROV's position in the next range assumes that the trajectory is linear, it is expected that the estimation error is greater in the circular and spiral paths than in the lawn mower pattern.

Table III was constructed based on this reasoning. In this table, the expected performances of the algorithm using the different approaches for the different paths are compared. The plus sign indicates the approach that is expected to produce the best performance for the path.

Table III: Expected performance of the approaches.

\begin{tabular}{c|c|c|c|c} 
Approach & Stationary & Lawn Mower & Circular & Spiral \\
\hline A & $=$ & - & + & + \\
B & $=$ & + & - & -
\end{tabular}

By analyzing the results on tables I and II, it can be verified that the simulation results follow the expected trends, with some variations that can be attributed to the measurement and estimation uncertainties.

\section{CONCLUSiOnS}

In this article, a guidance algorithm for an ASV to be used as a navigation aid for a UUV was developed. This work is based on treating the proposed guidance problem as a sensor-target placement geometry, which was resolved with two different metrics based on the analysis of the Fisher Information Matrix. Furthermore, the metrics used for guidance of the ASV also provide relevant information about the optimal initial positioning of the ASV relatively to the ROV.

The results of the performed tests indicate that the estimation error when estimating the position of an UUV using DR and a single moving beacon depend on the operating depth of the UUV and the maximum velocity of the ASV.

Analyzing the results of the performed tests, it can be verified that the positioning errors are low when compared to other similar works, such as [6] or [9]. However that direct comparison should only be taken as an indicator of the relevance of this approach to the problem, because of the simplicity of the simulation environment and the assumptions made about the reaching set of the surface vehicle.

Overall, it is possible to conclude that the the FIM is an appropriate tool for use in the guidance of a CNA surface vehicle.

\section{ACKNOWLEDGEMENTS}

This work is financed by the ERDF - European Regional Development Fund through the Operational Programme for Competitiveness and Internationalisation - COMPETE 2020 Programme within project «POCI-01-0145-FEDER-006961», and by National Funds through the FCT - Fundação para a Ciência e a Tecnologia (Portuguese Foundation for Science and Technology) as part of project UID/EEA/50014/2013.

\section{REFERENCES}

[1] B. Bingham, "Navigating Autonomous Underwater Vehicles," Underwater Vehicles, pp. 33-50, 2009.

[2] L. Paull, S. Saeedi, M. Seto, and H. Li, "AUV navigation and localization: A review," pp. 131-149, 2014.

[3] A. Bahr, J. J. Leonard, and M. F. Fallon, "Cooperative localization for autonomous underwater vehicles," International Journal of Robotics Research, vol. 28, no. 6, pp. 714-728, 2009.

[4] Y. T. Tan, R. Gao, and M. Chitre, "Cooperative path planning for rangeonly localization using a single moving beacon," IEEE Journal of Oceanic Engineering, 2014.

[5] D. Viegas, P. Batista, P. Oliveira, and C. Silvestre, "Position and velocity filters for asc/i-auv tandems based on single range measurements," Journal of Intelligent and Robotic Systems: Theory and Applications, 2014.

[6] I. Masmitja, P. Bouvet, S. Gomariz, J. Aguzzi, and J. del Rio, "Underwater mobile target tracking with particle filter using an autonomous vehicle,' OCEANS 2017 - Aberdeen, 2017.

[7] A. N. Bishop, B. Fidan, B. D. Anderson, K. Doğançay, and P. N Pathirana, "Optimality analysis of sensor-target localization geometries," Automatica, 2010

[8] B. M. Ferreira, A. C. Matos, H. S. Campos, and N. A. Cruz, "Localization of a sound source: optimal positioning of sensors carried on autonomous surface vehicles."

[9] M. Chitre, "Path planning for cooperative underwater range-only navigation using a single beacon," in IEEE 2010 International Conference on Autonomous and Intelligent Systems, AIS 2010, 2010. 